Time-lag cross-correlations in collective phenomena

This article has been downloaded from IOPscience. Please scroll down to see the full text article.

2010 EPL 9068001

(http://iopscience.iop.org/0295-5075/90/6/68001)

View the table of contents for this issue, or go to the journal homepage for more

Download details:

IP Address: 161.53.132.141

The article was downloaded on 02/07/2010 at 08:50

Please note that terms and conditions apply. 


\title{
Time-lag cross-correlations in collective phenomena
}

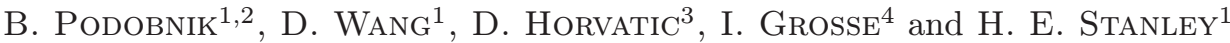 \\ ${ }^{1}$ Center for Polymer Studies and Department of Physics, Boston University - Boston, MA 02215, USA \\ ${ }^{2}$ Faculty of Civil Engineering, University of Rijeka - 51000 Rijeka, Croatia \\ ${ }^{3}$ Faculty of Science, University of Zagreb - 10000 Zagreb, Croatia \\ ${ }^{4}$ Martin Luther University, Institute of Computer Science - 06120 Halle, Germany, EU
}

received 18 May 2010; accepted in final form 11 June 2010

published online 30 June 2010

PACS 89.75. Da - Systems obeying scaling laws

PACS $89.90 .+n-$ Other topics in areas of applied and interdisciplinary physics

\begin{abstract}
We study long-range magnitude cross-correlations in collective modes of real-world data from finance, physiology, and genomics using time-lag random matrix theory. We find longrange magnitude cross-correlations i) in time series of price fluctuations, ii) in physiological time series, both healthy and pathological, indicating scale-invariant interactions between different physiological time series, and iii) in ChIP-seq data of the mouse genome, where we uncover a complex interplay of different DNA-binding proteins, resulting in power-law cross-correlations in $x_{i j}$, the probability that protein $i$ binds to gene $j$, ranging up to 10 million base pairs. In finance, we find that the changes in singular vectors and singular values are largest in times of crisis. We find that the largest 500 singular values of the NYSE Composite members follow a Zipf distribution with exponent $\approx 2$. In physiology, we find statistically significant differences between alcoholic and control subjects.
\end{abstract}

Copyright (C) EPLA, 2010

Introduction. - Many complex systems are part of even larger systems where the constituent complex systems mutually interact ${ }^{1}[1-3]$, giving rise to the appearance of "collective modes" [4-7]. Stochastic interactions among related systems are reflected by the presence of cross-correlations, and here we address the question of whether these cross-correlations in the collective modes exhibit power-law scale-invariant properties.

Zero-lag cross-correlations in the collective modes of empirical time series were analyzed by using random matrix theory (RMT) [6-9]. Recently, RMT became very successful in the analysis of cross-correlations between stock price changes, since cross-correlation matrices and associated covariance matrices play important roles in portfolio management $[10,11]$. A variety of studies reported the properties of the cross-correlation matrix $\mathrm{C}$ of price changes [6-14]. RMT enables a comparison between the cross-correlation matrix obtained from $N$ empirical time series each of length $T$ and a perfectly random matrix $W$, called a Wishart matrix, obtained from $N$ mutually uncorrelated time series each of length $T$ [15]. By analyzing cross-correlations between price changes of the members of the S\&P 500 index, it was found that $98 \%$ of the eigenvalue spectrum of the correlation

\footnotetext{
${ }^{1}$ Heartbeat interval time series, e.g., is one among many time series comprising the functioning human.
}

matrix C follows the Gaussian orthogonal ensemble of a perfectly random matrix $[7,8]$.

Recently, time-lag generalizations of RMT were proposed [16-21]. However, only short-range crosscorrelations were found. To quantify long-range collective movements in correlated data sets, we apply time-lag RMT (TLRMT) to the magnitude of three selected examples of real-world data: i) finance, ii) physiology, and iii) genomics.

Consider the $N$-variable time series $X=\left\{X_{i, t}: i=\right.$ $1, \ldots, N ; t=1, \ldots, T\}$ of length $T$, where $i$ indexes the series number, and $t$ denotes the time. The crosscorrelation matrices for this time series and for the magnitude time series are

$$
\begin{gathered}
C_{i j}(\Delta t) \equiv \frac{\left\langle X_{i, t} X_{j, t+\Delta t}\right\rangle-\left\langle X_{i, t}\right\rangle\left\langle X_{j, t+\Delta t}\right\rangle}{\sigma_{i} \sigma_{j}}, \\
\tilde{C}_{i j}(\Delta t) \equiv \frac{\left\langle\left|X_{i, t}\right|\left|X_{j, t+\Delta t}\right|\right\rangle-\left\langle\left|X_{i, t}\right|\right\rangle\left\langle\left|X_{j, t+\Delta t}\right|\right\rangle}{\tilde{\sigma}_{i} \tilde{\sigma}_{j}} .
\end{gathered}
$$

Here $\sigma_{i}, \sigma_{j}, \tilde{\sigma}_{i}$, and $\tilde{\sigma}_{j}$ denote the standard deviations of $X_{i, t}, X_{j, t+\Delta t},\left|X_{i, t}\right|$, and $\left|X_{j, t+\Delta t}\right|$, respectively, and $\langle\ldots\rangle$ denotes the time average.

In order to quantify cross-correlations for varying lags $\Delta t$, we compute the largest singular values $\lambda_{L}(\Delta t)$ and $\tilde{\lambda}_{L}(\Delta t)$ of the cross-correlation matrices $C(\Delta t)$ and $\tilde{C}(\Delta t)$ 
as functions of $\Delta t$ [20]. The squares of the non-zero singular values of $C$ are equal to the non-zero eigenvalues of $C C^{+}$or $C^{+} C$, where $C^{+}$denotes the transpose of $C$. In a singular value decomposition $C=U \Sigma V^{+}$the diagonal elements of $\Sigma$ are equal to the singular values of $C$. The columns of $U$ and $V$ are the left and the right singular vectors of the corresponding singular values. Consider a matrix $C$ with main diagonal elements $1 \mathrm{~s}$ and all offdiagonal elements being identical, i.e., $C_{i j}(\Delta t) \equiv C(\Delta t)$. Then we calculate the largest eigenvalue of $C C^{+}$(equal to $\left.\lambda_{L}^{2}(\Delta t)\right)$ and obtain

$$
\lambda_{L}^{2}(\Delta t)=1+(N-1)^{2} C(\Delta t)+2(N-1) C(\Delta t)^{2} .
$$

If $C_{i j}$ follows a power law $C_{i j}(\Delta t)=A(\Delta t)^{-\gamma}$, then for $\Delta t \gg 1, \lambda_{L}(\Delta t)=1+0.5 A(N-1)^{2}(\Delta t)^{-\gamma}$, where $A$ is a constant.

We find long-range cross-correlations in the following data series:

i) 1340 members $I_{i, t}$ of the New York Stock Exchange (NYSE) Composite. We analyze 1340 time series with 2172 daily records in the 8.7-year period, 2 January 2001 to 24 August 2009. We focus on the logarithmic change ("returns") defined by $R_{i, t} \equiv \ln \left(I_{i, t} / I_{i, t-1}\right)$, where $i$ denotes the index member [22], and $t$ stands for time in days. First we apply RMT to calculate $\lambda_{L}(\Delta t=$ $0)=392$ for returns $R_{i, t}$ and $\tilde{\lambda}_{L}(\Delta t=0)=359$ for volatilities $\left|R_{i, t}\right|$, and we find that both largest singular values are more than 100 times greater than expected for uncorrelated time series, indicating cross-correlations for $\Delta t=0$. Next, we apply TLRMT, and we plot $\lambda_{L}(\Delta t)$ and $\tilde{\lambda}_{L}(\Delta t)$ as a function of $\Delta t$ in fig. 1(a). We find long-range volatility cross-correlations, implying that $\left|R_{i, t}\right|$ affects $\left|R_{j, t+\Delta t}\right|(i \neq j)$ for $\Delta t>0$. Even though RMT shows that $\lambda_{L}(\Delta t=0)$ and $\tilde{\lambda}_{L}(\Delta t=0)$ are practically the same as found in ref. [6] for eigenvalues, it is TLRMT that shows that $\tilde{\lambda}_{L}(\Delta t)$ vs. $\Delta t$ decays more slowly than $\lambda_{L}(\Delta t)$, stating that volatility cross-correlations last longer than return cross-correlations, and we find that $\tilde{\lambda}_{L}(\Delta t)$ can be approximated by a power law $(\Delta t)^{-\gamma}$ with a scaling exponent of $\gamma=0.64 \pm 0.03$. Note that refs. $[23,24]$ reported power-law crosscorrelations between pairs of financial time series in magnitudes.

ii) Physiology Sleep Heart Health Study (SHHS) database $[25,26]$. For a single patient, we study 11 time series, $I_{i, t}$, among which are electroencephalography (EEG), heart rate, and electrooculogram. Here, $i=1, \ldots, 11$ denotes the index of the physiological time series, and $t$ denotes time in seconds. We show $\tilde{\lambda}_{L}(\Delta t)$ and $\lambda_{L}(\Delta t)$ in fig. 1(b), and we find that $\tilde{\lambda}_{L}(\Delta t) \sim(\Delta t)^{-\gamma}$ where $\gamma=0.06 \pm 0.01$. These findings indicate that TLRMT might be useful for distinguishing healthy from pathological behavior for
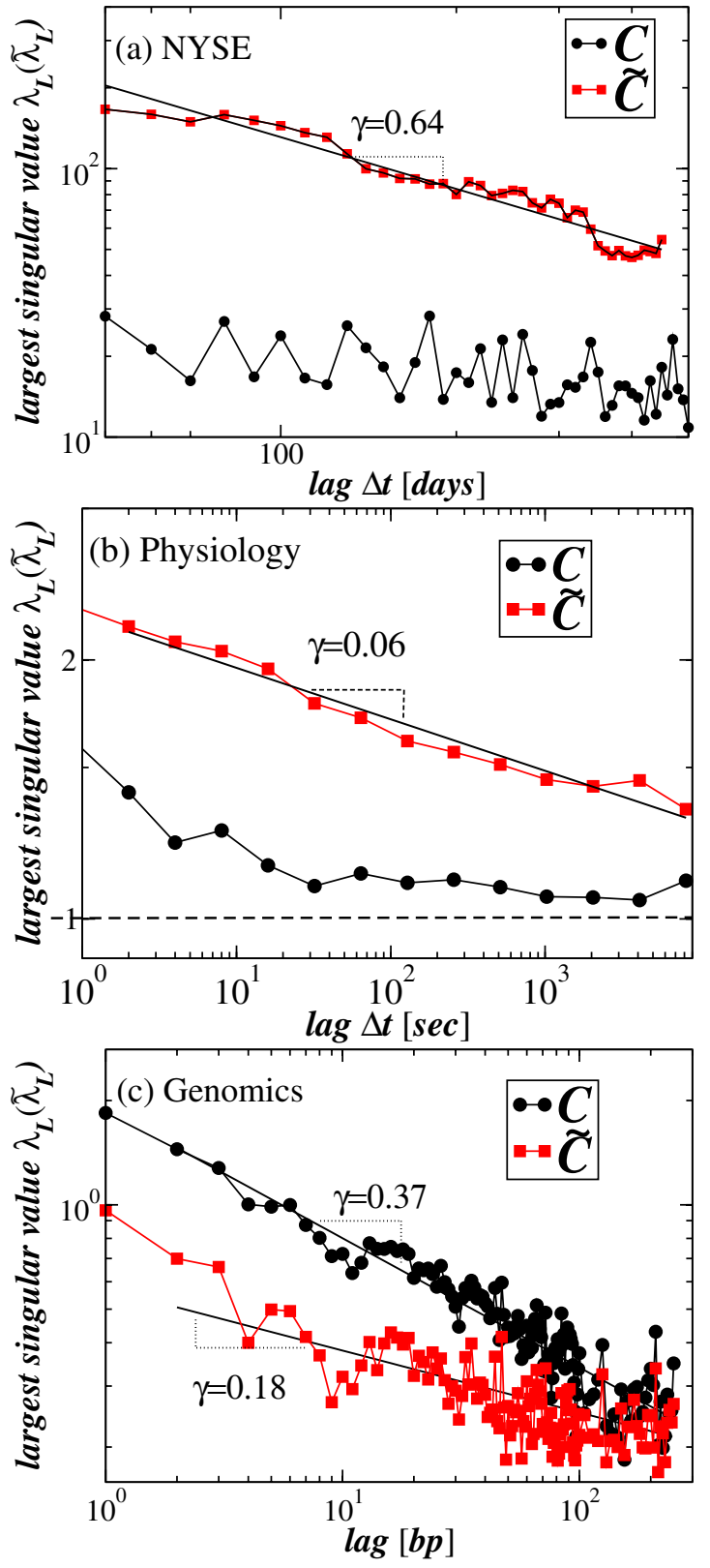

Fig. 1: (Colour on-line) Largest singular value vs. lag for (a) $N Y S E$ data. We find power-law volatility cross-correlations. With increasing $\Delta t, \lambda(\Delta t)$ decays more quickly than $\tilde{\lambda}(\Delta t)$. (b) Physiological data composed of $N=11$ time series, $I_{i, t}$, each of 32000 data points, where $i=1,2, \ldots, 11$. With increasing $\Delta t, \lambda$ decays more quickly than $\tilde{\lambda}(\Delta t)$, where $\tilde{\lambda}(\Delta t)$ decays as a power law. (c) Genomics data. For the largest mouse chromosome, $x_{i j}$ is the probability that protein $i$ binds to gene $j$. We find long-range power-law cross-correlations in $x_{i j}$.

multivariate correlated time series, the same as detrended fluctuation analysis (DFA) [27-30] for a single time series [5].

iii) ChIP-seq data of mouse chromosome 2 [31]. The binding affinity of 14 DNA-binding proteins to the DNA of mouse chromosome 2 was obtained by calculating 
the probability $x_{i j}$ that protein $i$ binds to gene $j$ on mouse chromosome 2 for all $i=1, \ldots, 14$ and $j=$ $1, \ldots, 1544$. We apply the DFA fluctuation function $F(n)[27-30]$ to these 14 numerical sequences (spatial not temporal) $x_{i j}$ of the same length, and we find that $F(n)$ can be approximated by a power law for all of the 14 numerical sequences, $F(n) \propto n^{\alpha}$. This power-law scaling of $F(n)$ with $n$ indicates the presence of long-range auto-correlations for the 14 individual sequences $x_{i j}$, and the average DFA scaling exponent $\alpha=0.69>0.5$ indicates that neighboring genes have a higher tendency to be both bound, or to be both unbound, by the same transcription factor than expected by chance. One possible interpretation of this finding is that there is some evolutionary advantage for a species if its genes whose promoters are bound by the same transcription factor are close to each other in the genome. The power-law scaling of $F(n)$ with $n$ states that this tendency does not decay exponentially with the chromosomal distance between the genes.

Next, we focus on nonequal-lag cross-correlations using the TLRMT approach. We show $\tilde{\lambda}_{L}(\Delta t)$ and $\lambda_{L}(\Delta t)$ in fig. $1(\mathrm{c})$, and we find approximate power-law cross-correlations with scaling exponents of $0.37 \pm 0.01$ and of $0.18 \pm 0.01$, respectively, implying that the binding or unbinding of protein $i$ to gene $j$ is influenced by the binding or unbinding of other proteins $i^{\prime}$ to gene $j$ or neighboring genes $j^{\prime}$. Interestingly, the neighborhood reaches up to $\left|j-j^{\prime}\right| \approx 100$ genes, corresponding to a chromosomal distance of approximately 10 million base pairs.

In order to investigate if TLRMT might be useful for prediction, we apply it to financial and genomics time series.

i) 88 companies that contribute to the $\mathrm{S} \& \mathrm{P} 500$ index in 2009 during the 26-year period 1983-2009. We apply TLRMT for each year, as in fig. 1(a), and we show $\tilde{\lambda}_{L}(\Delta t) v s$. year in fig. 2(a). We find pronounced peaks during the largest market shocks and economic crisis: the Black Monday, the Dot-com Bubble, and the 2008 crash. We study different time lags $\Delta t$, because the presence of cross-correlations for $\Delta t=0$ does not imply the presence of cross-correlations for $\Delta t \neq 0$, and indeed we find interesting differences when tuning the time lag $\Delta t \neq 0$. We investigate how $\tilde{\lambda}_{L}(\Delta t)$ changes over time lags (days) for different years, and we show $\tilde{\lambda}_{L}(\Delta t) v s$. time lag in fig. $2(\mathrm{~b})$. We find that $\tilde{\lambda}_{L}(\Delta t) \sim(\Delta t)^{-\gamma}$, where $\gamma$ varies from year to year and is greatest in times of crisis.

We calculate the absolute values of the 88 components $\left|u_{i}\left(\tilde{\lambda}_{L}(\Delta t)\right)\right|$ of the left-singular vectors corresponding to $\tilde{\lambda}_{L}(\Delta t)$ of the volatility time series in fig. 2(a). We show the mean $\mu$ and the standard
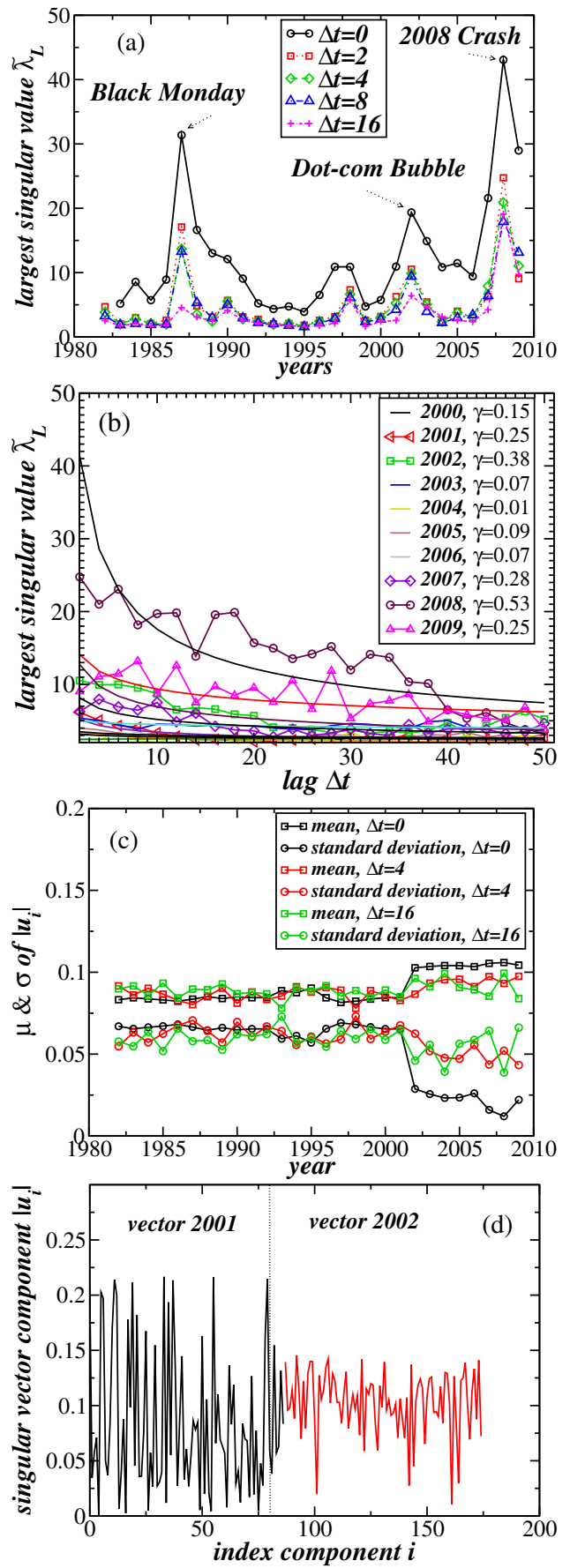

Fig. 2: (Colour on-line) Application of TLRMT in finance. For the longest $N=88$ members of the S\&P 500 index we calculate (a) $\tilde{\lambda}_{L}(\Delta t)$ vs. year for lags $0,2,4,8$, and 16 from top to bottom. TLRMT reveals pronounced peaks in times of crisis for varying $\Delta t$. (b) For each year we fit $\tilde{\lambda}_{L}(\Delta t) v s$. lag (in days) with a power law with exponent $\gamma$ that is especially increased in 2002 and 2008. (c) For each year considered, we calculate, for the largest singular value, the 88 singular vector components $u_{i}\left(\tilde{\lambda}_{L}(\Delta t)\right)$, where $\Delta t=0,4,16$. From the magnitudes, $\left|u_{i}\left(\tilde{\lambda}_{L}(\Delta t)\right)\right|$, we calculate the mean, $\mu$, and standard deviation, $\sigma$. In 2002, for $\Delta t=0, \mu(\sigma)$ suddenly increased (decreased). For $\Delta t=4,16$ we use left-singular vectors. (d) For $\Delta t=0$, sudden changes in $\left|u_{i}\left(\tilde{\lambda}_{L}(\Delta t)\right)\right|$ for two adjacent years are in agreement with (c). 


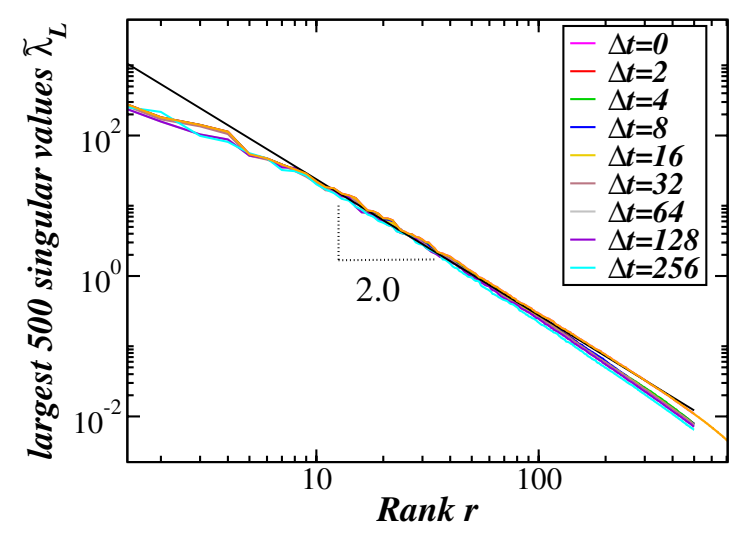

Fig. 3: (Colour on-line) Zipf distributions for the largest 500 singular values $\tilde{\lambda}(\Delta t)$ obtained from the volatility crosscorrelations among the NYSE Composite members. Zipf plots for different time lags practically overlap and can be well fit with a power law.

deviation $\sigma$ of the 88 values of $\left|u_{i}\left(\tilde{\lambda}_{L}(\Delta t)\right)\right|$ for each $\Delta t=0,4,16$ in fig. $2(\mathrm{c})$, and we find that $\mu$ suddenly increases in 2002, whereas $\sigma$ suddenly decreases. This finding can be partially explained by fig. $2(\mathrm{~d})$, where we find that $\left|u_{i}\left(\tilde{\lambda}_{L}(\Delta t)\right)\right|$ substantially change after the Dot-com Bubble crash in 2001.

In addition to the largest singular value $\tilde{\lambda}_{L}(\Delta t)$ shown in fig. 1(a), we calculate all singular values $\tilde{\lambda}(\Delta t)$ of the NYSE Composite. We show the rankordered distribution of the largest $500 \tilde{\lambda}(\Delta t)$ for each $\Delta t$ in fig. 3 . We find that the distributions for different $\Delta t$ practically overlap (power-law stability), and they can be approximated by a power law with exponent 2 . In comparison, probability density functions of returns exhibit power-law tails with exponent $\approx 4$ [32]. The first power law is accompanied by power-law volatility cross-correlations (fig. 1(a)), and the latter by power-law volatility autocorrelations [33-37].

ii) EEG time series. Reference [38] reported power-law auto-correlations in both EEG time series and their magnitudes, with different exponents for healthy subjects and subjects with Alzheimer's disease. Reference [23] reported power-law cross-correlations between pairs of EEG time series in magnitudes. Reference [39] reported that cross-correlations for $\Delta t=0$ calculated between pairs of EEG time series are inversely related to dissociative symptoms (psychometric measures) in 58 patients with paranoid schizophrenia.

Here we analyze multiple time series of EEG recordings of two groups of subjects: control and alcoholic [40]. These data arise from a study to examine EEG correlates of a genetic predisposition to alcoholism. Measurements were obtained from 64 electrodes placed on the scalp, sampled at $256 \mathrm{~Hz}(3.9 \mathrm{~ms}$
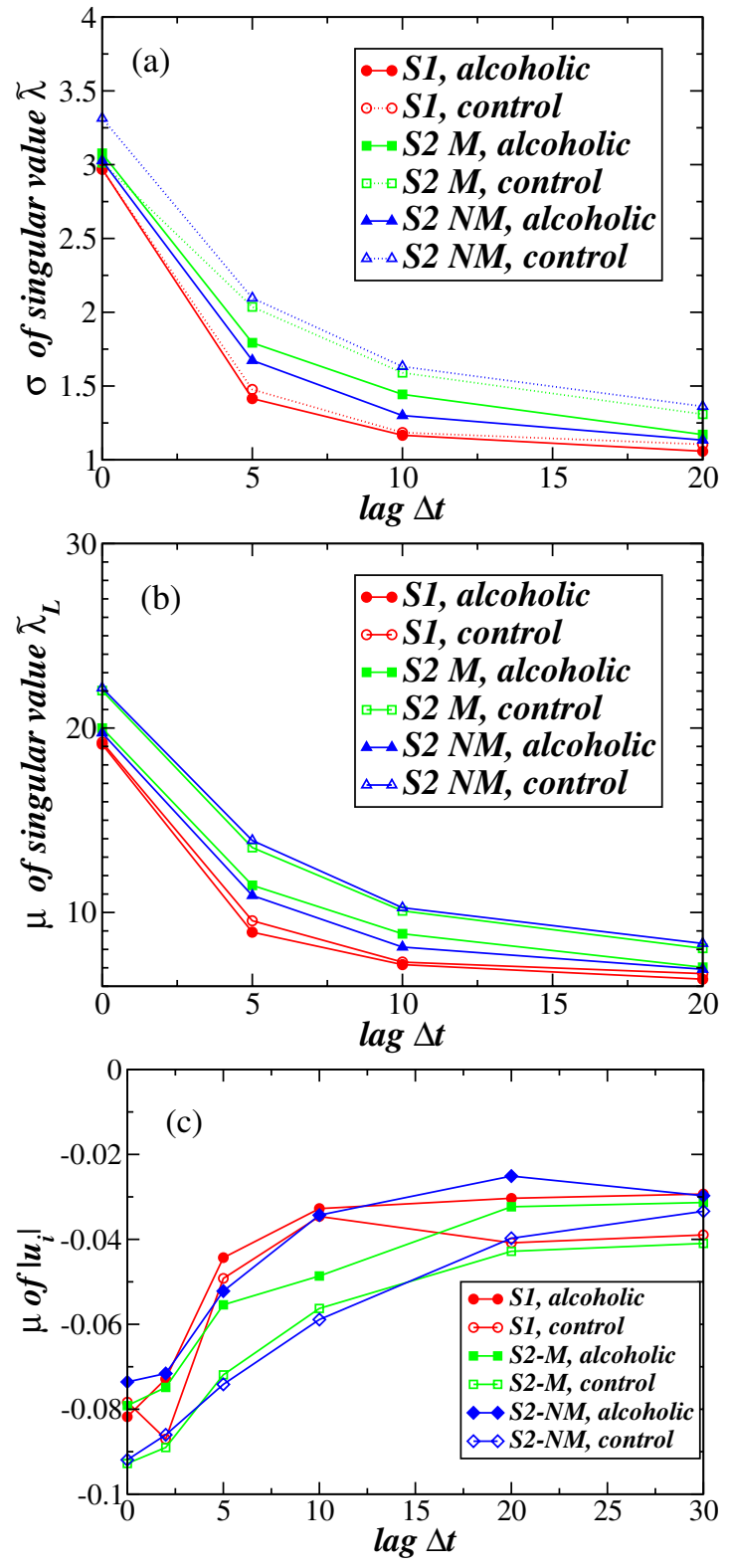

Fig. 4: (Colour on-line) Diagnostic application of TLRMT in physiology. (a) Standard deviation $\sigma$ of singular value (obtained from volatility cross-correlations) vs. lag for the EEG Dataset for alcoholic and control subjects. For S2-M and S2NM conditions (explained in the text), $\sigma$ for control subjects is larger than $\sigma$ for alcoholic subjects. (b) Mean $\mu$ of largest singular values vs. lag for alcoholic and control subjects. For S2$\mathrm{M}$ and S2-NM conditions, $\mu$ for control subjects is larger than $\mu$ for alcoholic subjects. (c) For each condition and alcoholic and control subjects, we show the mean $\mu$ of $\left|u_{i}\left(\tilde{\lambda}_{L}(\Delta t)\right)\right|$ of all right-singular vectors of the volatility time series. There is a significant difference between alcoholic and control subjects.

epoch) for 1 second. The electrodes were placed at standard sites (Standard Electrode Position Nomenclature, American Electroencephalographic Association 1990). Each of 122 subjects completed 120 trials. Each subject was exposed either to a single stimulus (S1) or to two stimuli (S2) which were 
different pictures of objects. If two stimuli are equal it is called a matched (S2-M) condition, whereas if two stimuli are different it is called a non-matched (S2-NM) condition.

We randomly choose 15 alcoholic and 15 control subjects. For a given time lag $(\Delta t=0,5,10,20)$ and a given condition (S1, S2-M, S2-NM), we calculate all singular values $\tilde{\lambda}(\Delta t)$ for each alcoholic subject and for each control subject. We calculate the standard deviations $\sigma$ of $\tilde{\lambda}(\Delta t)$ for each $\Delta t$, for each condition, and for alcoholic subjects and control subjects separately, and we show those standard deviations in fig. 4(a). We find that $\sigma$ for control subjects is greater than $\sigma$ for alcoholic subjects for each $\Delta t$ and each condition. An F-test confirms that the differences between alcoholic subjects and control subjects are statistically significant for the S2-M and the S2-NM condition. We show in fig. 4(b) the mean $\mu$ of $\tilde{\lambda}_{L}(\Delta t)$ for each $\Delta t$, for each condition, and for alcoholic subjects and control subjects separately. We find that $\mu$ for control subjects is substantially greater than for alcoholic subjects for the S2-M and the S2-NM conditions.

Next, for each condition and for alcoholic subjects and control subjects separately, we calculate the magnitudes of components $\left|u_{i}\left(\tilde{\lambda}_{L}(\Delta t)\right)\right|$ of all rightsingular vectors corresponding to $\lambda_{L}(\Delta t)$ of the volatility time series. For different conditions in fig. $4(\mathrm{c})$ we show the mean $\mu$ of $\left|u_{i}\left(\tilde{\lambda}_{L}(\Delta t)\right)\right|$ for varying $\Delta t$. For the $\mathrm{S} 2-\mathrm{M}$ and the $\mathrm{S} 2-\mathrm{NM}$ conditions, we find significant differences between alcoholic and control subjects. In case of left-singular vectors for small lags, for the same conditions, we find less substantial differences between alcoholic and control subjects.

In conclusion, cross-correlations are found in a number of studies including nanodevices [41-43], atmospheric geophysics [44], seismology [45], and finance [6,7,23,24, 46-55]. We study cross-correlations in both temporal and spatial collective modes using time-lag RMT (TLRMT). We find long-range cross-correlations in quite diverse systems, ranging in size from the Earth's atmosphere (a volume of approximately $5 \times 10^{18} \mathrm{~m}^{3}$ ) to microscopic systems such DNA sequences (a volume of $5 \times 10^{-18} \mathrm{~m}^{3}$ ), ranging from living to non-living systems, and ranging from physical to non-physical systems such as the financial market.

In genomics data, we find spatial cross-correlations corresponding to a chromosomal distance of $\approx 10$ million base pairs. In physiology, TLRMT reveals statistically significant difference in standard deviations and means of singular values between alcoholic and control subjects. In finance, by analyzing cross-correlations in the magnitudes of price fluctuations we find that the largest singular values and their singular vectors substantially change after the Dot-com Bubble crash in 2001. We also find that the largest 500 singular values of the NYSE Composite members follow a Zipf distribution. We find power-law decaying cross-correlations in the magnitudes of price fluctuations implying that large-magnitude fluctuations - commonly taken to quantify risk- created in one stock are transferred to other stocks, and that this impact lasts over many time scales. Such crosscorrelations are of potential interest in risk management. TLRMT reveals that cross-correlations are strongest during market crashes and global recessions.

Our findings are consistent with the interesting hypothesis that cross-correlations are ubiquitously present in many systems. Studying these cross-correlations is a necessary prerequisite for understanding them, and a deeper understanding of these cross-correlations enables a deeper understanding of these systems. A deeper understanding of these systems, in turn, enables improved clinical applications and increases our forecasting power. The TLRMT approach developed in statistical physics may contribute to this long-term goal and lead to potential advancements in diverse areas of science.

\section{REFERENCES}

[1] Meyers R. A. (Editor), Encyclopedia of Complexity and Systems Science (Springer) 2009.

[2] Christensen K. and Moloney N. R., Complexity and Criticality (Imperial College Press) 2010.

[3] Cohen R. and Havlin S., Complex Networks: Structure Robustness and Function (Cambridge University Press) 2010.

[4] Koscielny-Bunde E., Bunde A., Havlin S., Roman H. E., Goldreich Y. and Schellnhuber H. J., Phys. Rev. Lett., 81 (1998) 729.

[5] Ashkenazy Y., Ivanov P. Ch., Havlin S., Peng C.-K., Goldberger A. L. and Stanley H. E., Phys. Rev. Lett., 86 (2001) 1900.

[6] Laloux L., Cizeau P., Bouchaud J.-P. and Potters M., Phys. Rev. Lett., 83 (1999) 1467.

[7] Plerou V., Gopikrishnan P., Rosenow B., Amaral L. A. N. and Stanley H. E., Phys. Rev. Lett., 83 (1999) 1471.

[8] Plerou V., Gopikrishnan P., Rosenow B., Amaral L. A. N., Guhr T. and Stanley H. E., Phys. Rev. E, 65 (2002) 066126.

[9] Utsugi A., Ino K. and Oshikawa M., Phys. Rev. E, 70 (2004) 026110.

[10] Rosenow B., Gopikrishnan P., Plerou V. and Stanley H. E., Physica A, 314 (2002) 762.

[11] Rosenow B., Plerou V., Gopikrishnan P. and Stanley H. E., Europhys. Lett., 59 (2002) 500.

[12] Pan R. K. and Sinha S., Phys. Rev. E, 76 (2007) 046116.

[13] Nakayama Y. and Iyetomi H., Prog. Theor. Phys. Suppl., 179 (2009) 60.

[14] Shen J. and Zheng B., EPL, 86 (2009) 48005.

[15] Edelman A., SIAM J. Matrix Anal. Appl., 9 (1988) 543.

[16] Potters M., Bouchaud J.-P. and Laloux L., Acta Phys. Pol. B, 36 (2005) 2767. 
[17] Kwapien J., Drozdz S., Gorski A. Z. and Oswiecimka P., Acta Phys. Pol. B, 37 (2006) 3039.

[18] Mayya K. B. K. and AmritKar R. E., AAPPS Bull., 17 (2007) 96.

[19] Thurner S. and Biely C., Acta Phys. Pol. B, 38 (2007) 4111.

[20] Bouchaud J.-P., Laloux L., Miceli M. A. and Potters M., Eur. Phys. J. B, 55 (2007) 201.

[21] Nakamura T. and Small M., Physica A, 383 (2007) 96.

[22] http://ww. finance.yahoo.com.

[23] Podobnik B. and Stanley H. E., Phys. Rev. Lett., 100 (2008) 084102.

[24] Podobnik B., Horvatic D., Petersen A. M. and Stanley H. E., Proc. Natl. Acad. Sci. U.S.A., 106 (2009) 22079.

[25] http://www.physionet.org/physiobank/database/.

[26] Goldberger A. et al., Circulation, 101 (2000) E215.

[27] Peng C. K., Buldyrev S. V., Havlin S., Simons M., Stanley H. E. and Goldberger A. L., Phys. Rev. E, 49 (1994) 1685.

[28] Hu K., Ivanov P. Ch., Chen Z., Carpena P. and Stanley H. E., Phys. Rev. E, 64 (2001) 011114.

[29] Chen Z., Ivanov P. Ch., Hu K. and Stanley H. E., Phys. Rev. E, 65 (2002) 041107.

[30] Chen Z., Hu K., Carpena P., Bernaola-Galvan P., Stanley H. E. and Ivanov P. Ch., Phys. Rev. E, 71 (2005) 011104.

[31] Chen X. et al., Cell, 133 (2008) 1106.

[32] Gopikrishnan P., Plerou V., Amaral L. A. N., Meyer M. and Stanley H. E., Phys. Rev. E, 60 (1999) 5305.

[33] Ding Z. X., Granger C. W. J. and Engle R. F., J. Empir. Fin., 1 (1993) 83.

[34] Ding Z. X. and Granger C. W. J., J. Econom., 73 (1996) 185

[35] Liu Y., Cizeau P., Meyer M., Peng C.-K. and Stanley H. E., Physica A, 245 (1997) 437.

[36] Cizeau P., Liu Y., Meyer M., Peng C.-K. and Stanley H. E., Physica A, 245 (1997) 441.
[37] Liu Y., Gopikrishnan P., Cizeau P., Meyer M., Peng C.-K. and Stanley H. E., Phys. Rev. E, 60 (1999) 1390.

[38] Pan C. P., Zheng B., Wu Y. Z., Wang Y. and Tang X. W., Phys. Lett. A, 329 (2004) 130.

[39] Bob P., Susta M., Glaslova K. and Boutros N. N., Psychiatry Res., 177 (2010) 37.

[40] http://kdd.ics.uci.edu/databases/eeg/eeg.data.html.

[41] Samuelsson P., Sukhorukov E. V. and Buttiker M., Phys. Rev. Lett., 91 (2003) 157002.

[42] Cottet A., Belzig W. and Bruder C., Phys. Rev. Lett., 92 (2004) 206801.

[43] Neder I., Heiblum M., Mahalu D. and Umansky V., Phys. Rev. Lett., 98 (2007) 036803.

[44] Yamasaki K., Gozolchiani A. and Havlin S., Phys. Rev. Lett., 100 (2008) 228501.

[45] Campillo M. and Paul A., Science, 299 (2003) 547.

[46] TAKayasu M. and TAKayasu H., Statistical Physics and Economics (Cambridge University Press, Cambridge) 2010.

[47] Mantegna R. N., Eur. Phys. J. B, 11 (1999) 193.

[48] Kullmann L., Kertesz J. and Kaski K., Phys. Rev. E, 66 (2002) 026125.

[49] Matia K., Ashkenazy Y. and Stanley H. E., Europhys. Lett., 61 (2003) 422.

[50] Tumminello M., Aste T., Di Matteo T. and Mantegna R. N., Proc. Natl. Acad. Sci. U.S.A., 102 (2005) 10421.

[51] Mizuno T., Takayasu H. and Takayasu M., Physica A, 364 (2006) 336.

[52] Podobnik B., Fu D., Stanley H. E. and Ivanov P. Ch., Eur. Phys. J. B, 56 (2007) 47.

[53] Podobnik B., Horvatic D., Lam A., Stanley H. E. and Ivanov P. Ch., Physica A, 387 (2008) 3954.

[54] Arianos S. and Carbone A., J. Stat. Mech. (2009) P03037.

[55] Podobnik B., Grosse I., Horvatic D., Ilic S., Ivanov P. Ch. and Stanley H. E., Eur. Phys. J. B, 71 (2009) 243. 